
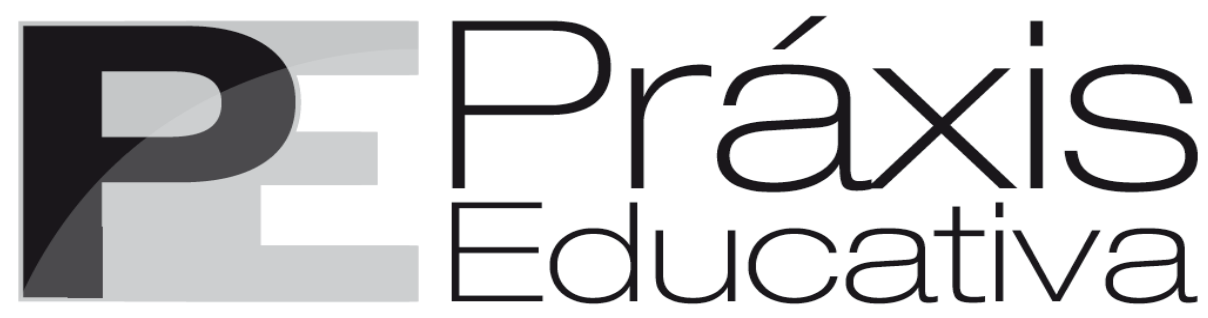

ISSN 1809-4031

eISSN 1809-4309

https://doi.org/10.5212/PraxEduc.v.16.16572.024

\title{
Estrategias de aprendizaje y creencias sobre el idioma inglés: una aproximación correlacional en estudiantes universitarios*
}

\section{Estratégias de aprendizagem e crenças sobre o idioma inglês: uma abordagem correlacional em estudantes universitários}

\section{Learning strategies and beliefs about English: a correlational approach among university students}

Juan Fernando Gómez**

iD https://orcid.org/0000-0003-1004-6793

Claudio Díaz Larenas ***

iD https://orcid.org/0000-0003-2394-2378

Walter Darién Gómez Torres ****

iD https:/ / orcid.org/0000-0002-7638-5811

Resumen: Este trabajo tiene como objetivos analizar las estrategias y las creencias sobre el aprendizaje de lenguas mediante el Strategy Inventory for Language Learning (SILL) y el Beliefs About Language Learning Inventory (BALLI) respectivamente, y examinar las correlaciones entre ambas variables, en una muestra de 303 estudiantes universitarios. Esta investigación es de tipo descriptivo correlacional, de corte transversal. Se usó la prueba U de Mann-Whitney para buscar diferencias en función del sexo y el test de Spearman para

\footnotetext{
* Este artículo es derivado del proyecto de investigación 'C reencias de los estudiantes del Tecnológico de Antioquia respecto al aprendizaje del inglés como lengua extranjera’, aprobado por el Comité de Bioética del Tecnológico de Antioquia - Institución universitaria. Dicho Proyecto fue financiado por el Comité para el Desarrollo de la Investigación (CODEI), según acta $\mathrm{N}^{\circ} 5$ de Reunión Ordinaria del 23/07/2015, centro de costos $\mathrm{N}^{\circ}$ 2060001073. Los instrumentos de recolección de datos se aplicaron en octubre de 2019.

** Tecnológico de Antioquia, Institución Universitaria (Colombia). Docente de tiempo completo de inglés del Departamento de Ciencias Básicas y Áreas Comunes. Candidato a Doctor en Ciencias de la Educación. E-mail: <jgomez2@tdea.edu.co>.

*** Universidad de Concepción (Chile). Profesor titular del Departamento de Currículum e Instrucción de la Facultad de Educación de la Universidad de Concepción. Doctor en Ciencias de la Educación. E-mail: <claudiodiaz@udec.cl>.

**** Tecnológico de Antioquia, Institución Universitaria (Colombia). Docente de tiempo completo, Coordinador del Departamento de Ciencias Básicas y Áreas Comunes. Candidato a Doctor en Humanidades y Artes. E-mail: <wgomez@tdea.edu.co>.
} 
Estrategias de aprendizaje y creencias sobre el idioma inglés: una aproximación correlacional...

encontrar correlaciones entre el SILL y el BALLI. Los resultados indicaron que las estrategias sociales se usan con mayor frecuencia, y se presentaron diferencias entre el sexo en las estrategias de memoria.

Palabras clave: Creencias. Correlaciones. Estrategias de aprendizaje. Competencia comunicativa.

Resumo: Este artigo tem como objetivo analisar estratégias e crenças de aprendizado de idiomas por meio de Strategy Inventory for Language Learning (SILL) e Beliefs About Language Learning Inventory (BALLI) respectivamente e examinar as correlações entre os dois, em uma amostra de 303 estudantes universitários. Esta pesquisa é descritiva, correlacional, transversal. O teste U de Mann-Whitney foi utilizado para encontrar diferenças de acordo com o sexo e o teste de Spearman, para encontrar correlações entre o SILL e o BALLI. Os resultados indicaram que as estratégias sociais são usadas com mais frequência e houve diferenças entre o sexo nas estratégias de memória.

Palavras-chave: Crenças. Correlações. Estratégias de aprendizagem. Competência comunicativa.

\begin{abstract}
This research intends to analyze the strategies and beliefs through the Strategy Inventory for Language Learning (SILL) and the Beliefs About Language Learning Inventory (BALLI), respectively, and examine the correlations between both instruments with a sample of 303 university students. This corresponds to a descriptive correlational study carried out under a transactional design. The Mann-Whitney $\mathrm{U}$ test was used to find gender-related differences and the Spearman's Correlation Test to find correlation between both SILL and BALLI. Results indicated that the category of 'social strategies' is used more often and gender differences in 'memory strategies' were also identified.
\end{abstract}

Keywords: Beliefs. Correlations. Learning strategies. Communicative competence.

\title{
Introducción
}

Desde la concepción del término 'competencia comunicativa', la comunicación oral se ha constituido en una gran prioridad en el proceso de enseñanza y aprendizaje de una lengua extranjera, según Demir et al. (2018), lo cual es consistente con los lineamientos del Marco Común Europeo de Referencia para las Lenguas del Consejo de Europa (2001). En el contexto global, el idioma inglés se ha convertido en la lengua más utilizada, ya que esta es requerida en los mercados laborales actuales y entrega a los graduados las herramientas y habilidades comunicativas necesarias para dicho propósito, según las consideraciones de Moslehifar y Ibrahim (2012). Li (2010) explica cómo diversos investigadores en el campo de la adquisición de una lengua han reconocido que existen variables que inciden en el aprendizaje, tales como la edad, la aptitud, la motivación, el estilo cognitivo, el estilo de aprendizaje, las creencias sobre el aprendizaje de una lengua y las estrategias de aprendizaje, estas dos últimas convertidas en temas que generan gran debate en la actualidad.

Podría afirmarse que los estudiantes tienen diferentes opiniones, concepciones y creencias sobre lo que se debe aprender, el mejor método a emplear, los recursos que deben utilizarse y los factores que influyen en el aprendizaje de una lengua extranjera. No obstante, "las creencias que tienen los estudiantes están basadas en experiencias previas y suposiciones que pueden ser correctas o incorrectas", según las consideraciones de Lightbown y Spada (2013, p. 97), y que evidentemente tendrán un efecto en el tipo de estrategias que deseen emplear para aprender una lengua extranjera.

En cuanto a las estrategias de aprendizaje, podrían definirse como aquellos recursos cognitivos que utilizan los estudiantes, los cuales, de acuerdo con Herrera (2009), incluyen la disposición, la motivación, las actividades de planificación, la dirección y el control que tiene el estudiante al momento de enfrentarse al aprendizaje. Al respecto, Feleciya et al. (2015) sustentan que dichas estrategias mejoran significativamente las habilidades comunicativas y el desempeño académico de los estudiantes.

En el contexto de la institución universitaria donde se realizó este estudio, en relación con el proceso de aprendizaje, se evidenció que un número importante de estudiantes carecen de

Práxis Educativa, Ponta Grossa, v. 16, e2116572, p. 1-17, 2021 Disponível em: < https://www.revistas2.uepg.br/index.php/praxiseducativa> 
estrategias que les permitan controlar, autoevaluar y facilitar su proceso de aprendizaje de la lengua extranjera. También existen estudiantes que tienen creencias sobre el aprendizaje de una segunda lengua que poco favorecen el aprendizaje del inglés.

Los objetivos de esta investigación se centraron en analizar las estrategias de aprendizaje para el aprendizaje de lenguas (SILL) y las creencias sobre el aprendizaje de una lengua (BALLI), y examinar las correlaciones entre ambas en una muestra de estudiantes universitarios que cursan la asignatura de inglés en los niveles A1 al B1.

\section{Marco teórico}

El Marco Común Europeo de Referencia para las Lenguas (MCER) es el estándar internacional que presenta las competencias que permiten a una persona comunicarse empleando diversos medios lingǘsticos según el Consejo de Europa (2001). En este marco se manifiesta que los estudiantes son los agentes que deben desarrollar las competencias y las estrategias, al igual que realizar tareas y actividades que les impliquen ser eficientes en diversas situaciones comunicativas. Lo anterior hace pensar en la importancia que tiene el desarrollo de la competencia estratégica como un elemento que puede permitir a un estudiante no solo suplir las necesidades lingüísticas que surgen en la comunicación, sino que también se convierta en un recurso metacognitivo que ayude al estudiante para que sea el gestor de su propio aprendizaje. La competencia estratégica es definida por Franco et al. (2009) como el conocimiento y la habilidad para emplear estrategias con el propósito de facilitar la comunicación y el aprendizaje de una lengua, lo que implica acciones conscientes y organizadas durante la interlocución (BARRIOS; MONTIJANO, 2017; GÓMEZ, 2017).

Las estrategias de aprendizaje son definidas por (OXFORD, 1990) como un conjunto de acciones determinadas, conductas, técnicas o pasos que emplean los estudiantes de manera intencionada con el propósito de mejorar, agilizar y facilitar el aprendizaje de una lengua extranjera; las estrategias también permiten el desarrollo de las habilidades comunicativas. Tri Febriani (2017) ha señalado que los estudiantes efectivos, al momento de aprender una lengua extranjera, no solo usan una estrategia; sino que una combinación de estrategias compatibles con su forma de aprender, y los requerimientos de la tarea que deben resolver. Por otra parte, no todas las estrategias son útiles para todos los estudiantes de una lengua extranjera (PAŠALIĆ, 2018).

Por ello, es importante que la presente investigación explore cuáles estrategias utilizan los estudiantes que son efectivos al momento de aprender una lengua extranjera (WAHDAH; AININ; HAMID, 2018). Oxford (1990) creó el cuestionario SILL para conocer la frecuencia en el uso de las estrategias de aprendizaje de las personas que aprenden una lengua extranjera, aspecto confirmado por diversos investigadores tales como Orrego y Díaz (2010) y Hakan et al. (2015), entre otros. La sigla SILL en inglés corresponde a Strategy Inventory for Language Learning y, en español, a Inventario de Estrategias para el Aprendiraje de Lenguas. Este instrumento contiene seis grupos de estrategias que comprenden los comportamientos de aprendizaje de una lengua extranjera (OXFORD; BURRY-STOCK, 1995; TUGRUL, 2012).

Por su parte, las creencias refieren a ideas o nociones predeterminadas sobre una serie de temas relacionados con el aprendizaje de una lengua (HAYATI, 2015). De hecho, los estudiantes de lenguas extranjeras llegan a la sala de clases con un conjunto de ideas específicas sobre la naturaleza y el proceso de aprendizaje, que influyen en los métodos e intentos por aprender un idioma extranjero (KOVACEVIC; AKBAROV, 2015). En este sentido, es esencial, comprender las creencias de los estudiantes para acercarse a un conocimiento más profundo de las estrategias de aprendizaje y las formas de planificación de la enseñanza de un idioma (CEN, 2019). Diversos

Práxis Educativa, Ponta Grossa, v. 16, e2116572, p. 1-17, 2021

Disponível em: < https://www.revistas2.uepg.br/index.php/praxiseducativa> 
Estrategias de aprendizaje y creencias sobre el idioma inglés: una aproximación correlacional...

investigadores han indicado que ciertas creencias son beneficiosas para el aprendizaje del estudiante, y otras podrían tener efectos negativos en el proceso (AHMADI et al., 2016). Por ejemplo, creencias positivas sobre el aprendizaje del inglés podrían compensar la falta de competencia comunicativa en el idioma extranjero del estudiante (ALI-ZAREI; RAHMANI, 2013).

En cuanto al BALLI, propuesto por Horwitz (1987), es un instrumento que permite obtener información sobre las creencias que tienen las personas frente al aprendizaje de una lengua. BALLI corresponde al acrónimo Beliefs About Language Learning Inventory, cuya traducción es Inventario de Creencias sobre el Aprendizaje de una Lengua, constituido por cinco dimensiones. Se ha seleccionado el BALLI en esta investigación, puesto que constituye una herramienta que se ha empleado por los investigadores en diferentes contextos, según autores como Sioson (2011), Azar y Saeidi (2013), Hapsari (2016), entre otros.

Azar y Saeidi (2013) investigaron las relaciones entre el uso de las SILL y las creencias del BALLI con una muestra de 200 estudiantes de inglés. Los resultados revelaron que se presentó una relación significativamente positiva entre el BALLI y el SILL. Además, las regresiones realizadas indicaron que las subescalas del BALLI correspondientes a las estrategias de aprendizaje y comunicación, y la aptitud para las lenguas extranjeras, se constituyen en predictores sustanciales sobre la frecuencia en el uso de todas las estrategias de aprendizaje. Por otro lado, se encontró que hay una correlación entre la totalidad del BALLI y las estrategias metacognitivas del SILL. Hayati (2015) investigó el rendimiento académico, las creencias y las estrategias de aprendizaje de estudiantes universitarios y descubrió que existía una correlación positiva, pero débil entre las creencias, y las estrategias, y el rendimiento académico de los participantes.

Por su parte, Hapsari (2016) examinó la relación entre el BALLI y el SILL con un grupo de 41 estudiantes de inglés de la Universidad Estatal de Surabaya, Indonesia. El propósito fue identificar la relación entre las creencias de los estudiantes frente al aprendizaje de una lengua y el uso de las estrategias de aprendizaje. Los resultados indicaron que se presentaron correlaciones entre el BALLI y el SILL en todas las categorías. De igual modo, se reportó que las estrategias afectivas y metacognitivas fueron las más utilizadas por los estudiantes, mientras que las de memoria fueron usadas en menor proporción. En la misma línea, Febriani (2017) propuso identificar el nivel de frecuencia en el empleo de las estrategias de aprendizaje a través del instrumento (BALLI), con un grupo de estudiantes universitarios en Indonesia. De igual forma, se examinó las relaciones entre ciertas creencias (BALLI) y algunas estrategias de aprendizaje. La frecuencia más alta se reportó en las estrategias metacognitivas. Las correlaciones de Pearson revelaron una relación negativa entre la estrategia metacognitiva y la aptitud, y se halló una correlación positiva entre las estrategias metacognitivas y la motivación.

Wahdah et al. (2018) intentaron establecer la correlación entre creencias y estrategias en estudiantes universitarios y observaron que las estrategias de aprendizaje de mayor uso eran las de tipo metacognitivo y afectivo; además, establecieron correlaciones significativas entre las estrategias de aprendizaje de los participantes y sus creencias sobre la lengua extranjera. Por su parte, Cen (2019) descubrió que estudiantes universitarios evidenciaban diferencias significativas en sus creencias y en las estrategias que utilizaban. A su vez, estableció una correlación moderada entre el uso global de estrategias y sus creencias del aprendizaje la lengua extranjera.

Práxis Educativa, Ponta Grossa, v. 16, e2116572, p. 1-17, 2021

Disponível em: < https://www.revistas2.uepg.br/index.php/praxiseducativa> 


\section{Metodología}

Este estudio corresponde a una investigación cuantitativa, de tipo descriptivo correlacional y de corte transversal. Los objetivos de esta investigación consistieron en analizar las estrategias de aprendizaje para el aprendizaje de lenguas y las creencias sobre el aprendizaje de una lengua y examinar las correlaciones entre ambas en una muestra de estudiantes universitarios que cursan la asignatura de inglés en los niveles A1 al B1.

Los datos fueron recolectados mediante dos cuestionarios auto-aplicados con respuestas anónimas (SILL y BALLI) de una muestra de 303 estudiantes, divididos entre 103 hombres (34,0\%) y 200 mujeres $(66,0 \%)$, quienes cursaban la asignatura de inglés en los niveles 1 al 6 , clasificados de la siguiente manera: curso inglés 1 y 2 (nivel A1 - 25,8\%), cursos 3 y 4 (niveles A2 - 48,9\%), y cursos 5 y 6 (nivel B1 -25,4\%). Los estudiantes de la muestra se encontraban realizando diferentes programas técnicos y tecnológicos en el Tecnológico de Antioquia, (TdeA, I.U), ubicado en la ciudad de Medellín, Colombia.

Los datos fueron obtenidos a través de dos instrumentos: el Inventario de Estrategias para el Aprendiraje de Lenguas (con su sigla en inglés de SILL) (OXFORD, 1990), versión 7.0 (ESL/EFL) y el Inventario de Creencias sobre el Aprendizaje de una Lengua (con su sigla en inglés de BALLI) (HORWITZ, 1987). En cuanto al instrumento SILL, se empleó la versión traducida y ajustada al contexto colombiano realizada por Orrego y Díaz (2010). Este cuestionario está conformado por 50 ítems (estrategias), que las personas utilizan para el aprendizaje de una lengua y comprende las siguientes categorías: 9 estrategias relacionadas con la memoria, 14 estrategias cognitivas, 6 estrategias de compensación, 9 estrategias metacognitivas, 6 estrategias afectivas y 6 estrategias sociales. Las unidades de valor que comprende cada ítem, se expresan en una escala Likert que indica la frecuencia de uso de las estrategias de aprendizaje, del 1 al 5. En esta escala, el 1 corresponde a "nunca", el 2 indica "casi nunca", el 3 es "algunas veces", el 4 representa "generalmente" y 5 corresponde a "siempre o casi siempre". Las escalas para comprender las frecuencias de uso del instrumento SILL son: porcentajes entre 3.5-5.0 son considerados como "alto uso de las estrategias", los valores entre 2.5-3.4 indican un "uso medio de las estrategias", y aquellos valores entre 1.0-2.4 se entienden como "bajo uso de las estrategias".

En lo referente al BALLI, se utilizó la versión traducida y adaptada al contexto colombiano realizada por Gómez (2018), la cual consta de 34 ítems distribuidos en cinco cuestionarios de la siguiente manera: 1. Aptitud para las lenguas extranjeras (8 ítems); 2. La dificultad del aprendizaje de una lengua extranjera (5 ítems); 3. La naturaleza del aprendizaje de lenguas (7 ítems); 4. Las estrategias de aprendizaje y comunicación ( 8 ítems); 5. Motivaciones y expectativas (6 ítems). El nivel de opinión de cada uno de los ítems se ponderó a través de la escala de Likert de la siguiente manera: 1 totalmente de acuerdo (TA), 2 de acuerdo (A), 3 neutral (N), 4 en desacuerdo (D) y 5 totalmente en desacuerdo (TD). Los datos se presentaron de manera porcentual en cada uno de los ítems que conforman las dimensiones.

Se empleó el software estadístico SPSS versión 24. Se realizó el análisis descriptivo de los datos y se continuó con las pruebas de normalidad (Kolmogorov-Smirnov) para todas las variables (SILL y BALLI). Debido a que algunas de ellas no presentaron distribuciones normales, los análisis posteriores fueron con estadística no paramétrica. Para establecer si existían o no diferencias estadísticamente significativas $(\mathrm{p}<.05)$ en las puntuaciones de las variables del SILL y el BALLI en función del sexo, se empleó la prueba de U de Mann-Whitney por tratarse de dos muestras independientes. Para determinar si hay o no diferencias estadísticamente significativas $(p<.05)$ entre estas variables, se aplicó el test de Spearman para correlaciones bivariadas. Los coeficientes de confiabilidad de los cuestionarios fueron confirmados por medio del test Alfa de Cronbach, el cual 
Estrategias de aprendizaje y creencias sobre el idioma inglés: una aproximación correlacional...

indicó un valor de 0.945 para el SILL y 0.644 para el BALLI. Esto indica que la consistencia interna de los ítems del BALLI es aceptable y la del SILL es alta.

\section{Resultados}

Los resultados obtenidos a través de la estadística descriptiva revelaron que los participantes de este estudio se encuentran en un rango medio en cuanto a la frecuencia de uso de las estrategias de aprendizaje presentadas en el SILL $(\mathrm{M}=2.82$ / DS = 0.69). Además, se observó que todas las medias correspondientes a cada categoría están dentro de un rango medio. El análisis estadístico de cada categoría indicó que las estrategias sociales son las que se emplean con mayor frecuencia $(\mathrm{M}=3.08$ / DS = 0.77), seguidas de las de compensación $(\mathrm{M}=2.97$ / DS = 0.66) (Véase Tabla 1 más abajo). Esto significa que los estudiantes de este estudio prefieren emplear las estrategias sociales en mayor proporción para su aprendizaje del inglés. Este hallazgo es distante de la investigación realizada por Sioson (2011), ya que la media más alta se encontró en las estrategias cognitivas; y en el estudio de Hapsari (2016) resultaron ser las estrategias de compensación. Por otro lado, se halló una coincidencia con los estudios de Azar y Saeidi (2013), y Wahdah et al. (2018), dado que se descubrió que las estrategias metacognitivas son las más usadas por las muestras de sus estudios.

Los resultados anteriores podrían explicarse de la siguiente manera: primero, las estrategias sociales implican que el aprendizaje se origina a través de la interacción con otras personas, lo que implica señalar a un interlocutor que disminuya la velocidad del discurso o solicitarle la repetición del mensaje puesto que no fue lo suficientemente claro. De igual forma, en esta categoría se encuentra la opción de solicitar al docente la corrección de algo en particular al momento de hablar. Posiblemente, los estudiantes de esta muestra han considerado que esta categoría cobra una importancia alta debido a que tienen la necesidad de aprender, trabajar en equipo y requieren la intervención del docente, lo cual podría explicar la mayor puntuación, lo que indica las preferencias por las estrategias sociales.

Luego, se encontró que las estrategias de compensación son las que más se emplean después de las sociales. Estas hacen referencia a las acciones alternativas que se ejecutan para suplir la falta de conocimiento al comunicarse en una lengua extranjera. Esto puede sugerir que la media resultante en esta categoría es un indicador de las deficiencias lingüísticas que tiene esta muestra; por lo tanto, se hace necesario hacer uso frecuente de dichas estrategias para expresar las ideas, acudiendo a recursos como inventar palabras, adivinar el significado, cambiar al español y usar gestos. 
Tabla 1 - Comparación con las medias internacionales para las categorías SILL

\begin{tabular}{|c|c|c|c|c|c|c|}
\hline $\begin{array}{l}\text { Medias } \\
\text { SILL }\end{array}$ & $\begin{array}{c}\text { Estrategias de } \\
\text { memoria }\end{array}$ & $\begin{array}{l}\text { Estrategias } \\
\text { cognitivas }\end{array}$ & $\begin{array}{l}\text { Estrategias de } \\
\text { compensación }\end{array}$ & $\begin{array}{c}\text { Estrategias } \\
\text { metacognitivas }\end{array}$ & $\begin{array}{c}\text { Estrategias } \\
\text { afectivas }\end{array}$ & $\begin{array}{c}\text { Estrategias } \\
\text { sociales }\end{array}$ \\
\hline $\begin{array}{l}\text { Sioson } \\
(2011)\end{array}$ & $\begin{array}{c}3.02 \\
\text { (DS } 0.54)\end{array}$ & $\begin{array}{c}3.38 \\
\text { (DS } 0.63)\end{array}$ & $\begin{array}{c}3.16 \\
\text { (DS } 0.70)\end{array}$ & $\begin{array}{c}3.55 \\
\text { (DS } 0.76)\end{array}$ & $\begin{array}{c}3.10 \\
\text { (DS } 0.72)\end{array}$ & $\begin{array}{c}3.25 \\
\text { (DS 0.72) }\end{array}$ \\
\hline $\begin{array}{c}\text { Azar y } \\
\text { Saeidi } \\
(2013)\end{array}$ & $\begin{array}{c}2.94 \\
\left(\mathrm{DS}{ }^{*}\right)\end{array}$ & $\begin{array}{c}3.18 \\
\left(\mathrm{DS}{ }^{*}\right)\end{array}$ & $\begin{array}{c}3.32 \\
\left(\mathrm{DS}{ }^{*}\right)\end{array}$ & $\begin{array}{c}3.82 \\
(\mathrm{DS} *)\end{array}$ & $\begin{array}{c}3.19 \\
(\mathrm{DS} *)\end{array}$ & $\begin{array}{c}3.41 \\
(\mathrm{DS} *)\end{array}$ \\
\hline $\begin{array}{l}\text { Hapsari } \\
\text { (2016) }\end{array}$ & $\begin{array}{c}2.63 \\
\text { (DS } 0.58)\end{array}$ & $\begin{array}{c}2.77 \\
\text { (DS } 0.54)\end{array}$ & $\begin{array}{c}2.95 \\
\text { (DS } 0.66)\end{array}$ & $\begin{array}{c}3.28 \\
\text { (DS } 0.69)\end{array}$ & $\begin{array}{c}3.29 \\
\text { (DS } 0.63)\end{array}$ & $\begin{array}{c}2.72 \\
\text { (DS } 0.66)\end{array}$ \\
\hline $\begin{array}{l}\text { Febriani } \\
\text { (2017) }\end{array}$ & $\begin{array}{c}3.1 \\
(\mathrm{DS} *)\end{array}$ & $\begin{array}{c}3.7 \\
(\mathrm{DS} *)\end{array}$ & $\begin{array}{c}3.8 \\
(\mathrm{DS} *)\end{array}$ & $\begin{array}{c}3.9 \\
(\mathrm{DS} *)\end{array}$ & $\begin{array}{c}3.2 \\
(\mathrm{DS} *)\end{array}$ & $\begin{array}{c}3.4 \\
(\mathrm{DS} *)\end{array}$ \\
\hline $\begin{array}{c}\text { Wahdah } \\
\text { et al. } \\
(2018)\end{array}$ & $\begin{array}{c}2.56 \\
(\mathrm{DS} *)\end{array}$ & $\begin{array}{c}2.12 \\
(\mathrm{DS} *)\end{array}$ & $\begin{array}{c}2.13 \\
(\mathrm{DS} *)\end{array}$ & $\begin{array}{c}2.68 \\
(\mathrm{DS} *)\end{array}$ & $\begin{array}{c}2.67 \\
(\mathrm{DS} *)\end{array}$ & $\begin{array}{c}2.42 \\
(\mathrm{DS} *)\end{array}$ \\
\hline $\begin{array}{c}\text { TdeA- } \\
\text { I.U. } \\
(2020)\end{array}$ & $\begin{array}{c}2.80 \\
\text { (DS } 0.66)\end{array}$ & $\begin{array}{c}2.74 \\
\text { (DS } 0.65)\end{array}$ & $\begin{array}{c}2.97 \\
\text { (DS } 0.66)\end{array}$ & $\begin{array}{c}2.84 \\
\text { (DS } 0.74)\end{array}$ & $\begin{array}{c}2.52 \\
\text { (DS } 0.69)\end{array}$ & $\begin{array}{c}3.08 \\
\text { (DS } 0.77 \text { ) }\end{array}$ \\
\hline
\end{tabular}

Fuente: Elaboración propia.

En cuanto al BALLI, los resultados revelaron que los participantes del estudio tienen creencias que promueven el aprendizaje del inglés y se presentaron otras que, por el contrario, generan barreras que lo dificultan. Dentro de las cinco dimensiones analizadas, la comparación con las medias internacionales (véase Tabla 2) presentó los siguientes resultados: las creencias que conformaron las "Motivaciones y expectativas" alcanzaron el puntaje mayor $(\mathrm{M}=4.14$ / SD = $0.69)$, seguido de "La naturaleza del aprendizaje de lenguas" $(\mathrm{M}=3.71 / \mathrm{DS}=0.66)$ y en tercer lugar las "Estrategias de aprendizaje y comunicación" $(\mathrm{M}=3.44 / \mathrm{DS}=0.74)$. Esto indica que estos participantes consideran que la motivación es el factor de mayor relevancia en el aprendizaje del inglés, lo cual es afín con la investigaciones realizadas por Meshkat y Saeb (2012), Azar y Saeidi (2013) y Wahdah et al. (2018).

La dimensión correspondiente a la aptitud para las lenguas extranjeras indicó que un 77,2\% de la muestra estuvo de acuerdo con el hecho de que para los niños aprender una lengua extranjera es más fácil que para los adultos, lo que lleva a pensar que es posible que estos participantes piensan que los niños tienen ciertas habilidades que los adultos no poseen. El 68,6\% estuvieron de acuerdo con que algunas personas tienen habilidades especiales. Ambos porcentajes sugieren que estos participantes están dejando a un lado el uso de estrategias metacognitivas para el aprendizaje. El $31,4 \%$ atribuye el aprendizaje del inglés a la inteligencia, estos resultados son afines con los hallazgos de Meshkat y Saeb (2012). Los datos anteriores podrían sugerir que existen creencias que inciden en la actitud de las personas y reducen de cierto modo la motivación e influyen en la elección de las estrategias de aprendizaje.

En la dimensión de la dificultad del aprendizaje de una lengua extranjera, un 32,0\% manifestó estar de acuerdo con el hecho de que el inglés es un idioma difícil de aprender. Un 38,6\% se muestra pesimista frente al tiempo que requiere para aprender inglés en este contexto, el cual es entre 4 y 5 años, y para el 30,0\% es entre 1 y 2 años. En lo referente a las habilidades, el 47,5\% está de acuerdo con la aseveración de que hablar es más fácil que escuchar, y el 48,5\% definió que leer es más fácil que escribir. A pesar del optimismo que estos estudiantes manifestaron tener en la dimensión correspondiente a las motivaciones y expectativas, en esta misma se evidenciaron creencias que se constituyen en fuentes de dificultad o que podrían facilitar el aprendizaje. Estos resultados son similares a los de Meshkat y Saeb (2012), y Hapsari (2016). Sería posible pensar que las dificultades frente al aprendizaje del inglés y la motivación, podrían estar asociadas, y las 
Estrategias de aprendizaje y creencias sobre el idioma inglés: una aproximación correlacional...

percepciones frente a la dificultad podrían de cierto modo influir en el nivel de confianza de un estudiante, sobre todo al emplear el idioma con fines comunicativos. De hecho, podría afirmarse que los estudiantes que consideran difícil al idioma extranjero, tienden a sentirse menos motivados y esta podría ser la explicación para los porcentajes bajos correspondientes a la dimensión de las estrategias de aprendizaje y comunicación.

Frente a la dimensión de la naturaleza del aprendizaje de lenguas, un 32,3\% consideró que es importante tener conocimientos sobre los aspectos culturales del idioma para hablar inglés, y un $79,9 \%$ piensa que un país de habla inglesa es el lugar adecuado para aprenderlo. Para el $82,2 \%$ de los participantes, el vocabulario es la parte más esencial en el aprendizaje, el 65.0\% definió que el aprendizaje del inglés requiere de condiciones especiales, el 39.3\% favoreció la traducción del español al inglés como el aspecto de mayor trascendencia, el $82.2 \%$ pensó que la tipología sintáctica del español es diferente a la del inglés, y el 57.1\% reconoció estar de acuerdo con el hecho de que la parte más importante del aprendizaje del inglés es la gramática. Estos resultados están en la misma línea del estudio de Meshkat y Saeb (2012), y sugieren, de algún modo, que los encuestados están a favor del método de "gramática y traducción", en el cual, según Tugrul (2013), se identifican las diferencias y similitudes entre la L1 y L2 para comprender mejor el nuevo sistema lingüístico. Según Alcalde (2011), se enseña la lengua extranjera por medio de la lengua materna (reglas gramaticales y traducción directa e inversa).

En la dimensión de las estrategias de aprendizaje y comunicación, el 86.5\% de los encuestados optó por creer que se debe hablar con una pronunciación excelente y un $68.0 \%$ respaldó la idea de que se deben tomar riesgos al emplear la lengua extranjera. Por otro lado, un $57.1 \%$ piensa que no es adecuado emplear la estrategia de adivinar palabras según el contexto. Esto permitiría concluir que este último porcentaje de estudiantes no es consciente de la importancia que tiene la estrategia de inferir vocabulario del contexto en el que se presenta, como una de las maneras más efectivas de aprenderlo, según Tugrul (2012).

Además, es inevitable que los estudiantes cometan errores, debido a que esto es parte del proceso de aprendizaje de una lengua extranjera, aunque un 43,6\% de la muestra consideró que no se le debe permitir cometer errores a los estudiantes principiantes. Casi la totalidad de la muestra, $94.7 \%$, respaldó la idea de que se debe repetir y practicar mucho para aprender inglés y un $75.2 \%$ definió que los recursos audiovisuales son de alta importancia para el aprendizaje. Un $43.6 \%$ desea ser corregido al hablar y un 38.3\% siente vergüenza al hablar en inglés. Es evidente que el habla es una habilidad productiva que consiste en emplear reglas gramaticales, elementos cohesivos, vocabulario y aspectos fonológicos que son necesarios para expresar correctamente nuestros pensamientos, entre otros mensajes. Por lo tanto, se podría atribuir este último porcentaje a la ansiedad que dicha actividad genera y que se manifiesta con aspectos como: aumento de la presión arterial, sudoración, evitar mirar a las personas que se encuentran alrededor. Según lo indica Ortega (2013, p. 200), la ansiedad "genera olvido de aquello que se preparó con anterioridad y hasta bloqueos, lo cual le ocurre a los estudiantes que presentan un nivel de ansiedad más alto".

Finalmente, en la dimensión de las motivaciones y expectativas, un $74.9 \%$ de los participantes creen que lograrán hablar inglés muy bien, un $75.9 \%$ confirma que el aprendizaje de esta lengua les posibilitará entender la cultura norteamericana, el 94,1\% tuvo la convicción de que las oportunidades laborales se ampliarán si lo aprenden, un $46.5 \%$ desea aprender inglés porque quiere tener amigos norteamericanos y el 97,7\% tiene el deseo de aprender a hablar inglés muy bien. Estos resultados sugieren que estos participantes tienen una motivación instrumental alta, que se refleja en sus horizontes laborales, en contraste con una motivación más baja, evidenciada en su deseo de ser parte de una comunidad de hablantes angloparlantes. Las consideraciones anteriores son similares a los resultados obtenidos en los estudios de Meshkat y Saeb (2012), Azar

Práxis Educativa, Ponta Grossa, v. 16, e2116572, p. 1-17, 2021

Disponível em: < https://www.revistas2.uepg.br/index.php/praxiseducativa> 
y Saeidi (2013), Hapsari (2016) y Wahdah et al. (2018). Aunque los porcentajes señalaron que estos estudiantes tienen una motivación alta, esto no es un indicador que demuestre la dedicación y el esfuerzo para el estudio de esta lengua.

Tabla 2 - Comparación con las medias internacionales para las dimensiones del BALLI

\begin{tabular}{|c|c|c|c|c|c|}
\hline $\begin{array}{l}\text { Medias } \\
\text { BALLI }\end{array}$ & $\begin{array}{l}\text { Aptitud para } \\
\text { las lenguas }\end{array}$ & $\begin{array}{l}\text { Dificultad del } \\
\text { aprendizaje de } \\
\text { una lengua }\end{array}$ & $\begin{array}{l}\text { Naturaleza del } \\
\text { aprendizaje } \\
\text { de una lengua }\end{array}$ & $\begin{array}{l}\text { Estrategias de } \\
\text { aprendizaje y } \\
\text { comunicación }\end{array}$ & $\begin{array}{c}\text { Motivaciones y } \\
\text { expectativas }\end{array}$ \\
\hline $\begin{array}{l}\text { Sioson } \\
(2011)\end{array}$ & 2.37 & 2.46 & 2.04 & 2.42 & 1.49 \\
\hline DS & 0.45 & 0.56 & 0.58 & 0.50 & 0.50 \\
\hline $\begin{array}{c}\text { Meshkat y } \\
\text { Saeb } \\
(2012) \\
\end{array}$ & 3.43 & 3.61 & 3.75 & 3.76 & 4.08 \\
\hline DS & 1.15 & 1.14 & 1.12 & 1.16 & 1.16 \\
\hline $\begin{array}{l}\text { Azar y } \\
\text { Saeidi } \\
(2013)\end{array}$ & 3.30 & 3.21 & 3.28 & 3.62 & 4.07 \\
\hline DS & $*$ & $*$ & $*$ & $*$ & $*$ \\
\hline $\begin{array}{l}\text { Wahdah } \\
\text { et al. } \\
(2018)\end{array}$ & 3.43 & 3.34 & 3.51 & 3.36 & 3.58 \\
\hline DS & $*$ & $*$ & $*$ & $*$ & $*$ \\
\hline $\begin{array}{l}\text { TdeA- } \\
\text { I.U. } \\
(2020)\end{array}$ & 3,36 & 3,21 & 3,71 & 3,44 & 4,14 \\
\hline DS & 0.66 & 0.65 & 0.66 & 0.74 & 0.69 \\
\hline
\end{tabular}

Fuente: Elaboración propia.

En lo concerniente al sexo, la prueba U de Mann-Whitney demostró que solo se presentaron diferencias significativas en la categoría de las estrategias de memoria correspondientes al SILL, con un resultado de 8878,5 y un p-valor $=0.049 *$ (Véase Tabla 3). Este hallazgo indica que las estrategias de memoria son empleadas con mayor frecuencia en las mujeres que en los hombres. La prueba U de Mann-Whitney también definió que no se presentaron diferencias en ninguna de las dimensiones BALLI con relación al sexo. En lo concerniente al SILL, estos resultados distan de los hallazgos de Zhou (2010), quien encontró que las mujeres usan más las estrategias de compensación y García Herrero (2012) comprobó que son las de memorización, cognitiva y metacognitiva aquellas que las mujeres emplean con mayor frecuencia; Hakan et al. (2015) identificaron que son las de compensación las de mayor uso en las mujeres, mientras que Chang y Shen (2010) y Risueño et al. (2016), no encontraron ninguna diferencia estadísticamente significativa en el empleo de las estrategias de aprendizaje entre ambos sexos. En cuanto al BALLI, Chang y Shen (2010) descubrieron diferencias en la dimensión de las motivaciones y expectativas, Daif-Allah (2012) las detectó en las dimensiones de aptitud, estrategias comunicativas y en las motivaciones y expectativas. Por su parte, Mudra (2016) no encontró ninguna diferencia.

A pesar de que la prueba aplicada para identificar diferencias entre mujeres y hombres no encontró resultados estadísticamente significativos, se observa que todas las medias de las mujeres son superiores a las de los hombres en todas las categorías del SILL. Las puntuaciones más altas en las mujeres corresponden a las estrategias sociales $(M=3.00)$ y las de compensación $(M=3.10)$, lo que muestra que están en un rango medio de uso. La revisión bibliográfica realizada por Nahavandi y Mukundan (2014) reportó que se presenta un mayor uso de estrategias de aprendizaje de una lengua en las mujeres. No obstante, esto no es completamente consistente, ya que en los resultados de esta investigación solamente se presentó diferencia en la categoría de las estrategias de memoria en favor de las mujeres. Podría deducirse de estos resultados que las mujeres que 
Estrategias de aprendizaje y creencias sobre el idioma inglés: una aproximación correlacional...

conformaron esta muestra, emplean con mayor frecuencia diversas estrategias de memoria que corresponden a diferentes recursos y técnicas que permiten almacenar y organizar la información durante el aprendizaje del inglés. Esta categoría indica que las mujeres tienen la habilidad de hacer conexiones entre los nuevos conocimientos y los existentes, crear enlaces visuales y auditivos, construir imágenes mentales, recordar y hacer listados de vocabulario y refrescar conocimientos mediante el repaso.

Esta diferencia podría ser explicada a partir de la superioridad que evidencian las mujeres en la ejecución de tareas que implican la memoria y el aprendizaje verbal, de acuerdo con la investigación realizada por Navarro et al. (2014). Al respecto, Nahavandi y Mukundan (2014) afirman que, de acuerdo con algunos investigadores, se presentan diferencias en el uso de las estrategias de aprendizaje debido a predisposiciones genéticas y a cierto tipo de comportamientos relacionados con el pensamiento reflexivo, el cual hace referencia a la metacognición o socialización.

Otro aspecto a considerar, se refiere a la naturaleza y la personalidad de las mujeres, dado que de acuerdo con López Rúa (2006), ellas presentan una mayor motivación para explorar diversas estrategias de aprendizaje en comparación con los hombres. Este investigador también sustenta que existen diferencias entre ambos sexos en lo concerniente a los intereses, habilidades y condiciones sociales, las cuales también podrían causar que las mujeres las empleen más frecuentemente.

Tabla 3 - Diferencias entre el sexo para las categorías SILL y las creencias BALLI

\begin{tabular}{|l|c|c|c|c|c|c|}
\hline \multirow{2}{*}{$\begin{array}{c}\text { Categorías SILL y Dimensiones } \\
\text { BALLI }\end{array}$} & \multicolumn{2}{c|}{$\begin{array}{c}\text { Hombres } \\
(\mathbf{n = 1 0 3})\end{array}$} & \multicolumn{2}{c|}{ Mujeres (n=200 } & & \\
\cline { 2 - 7 } & $\boldsymbol{M}$ & $\mathbf{D S}$ & $\boldsymbol{M}$ & $\mathbf{D S}$ & $\boldsymbol{U}$ & $\boldsymbol{p}$ \\
\hline Estrategias de memoria & 2,70 & 0,68 & 2,85 & 0,64 & 8878,5 & $0,049 *$ \\
\hline Estrategias cognitivas & 2,66 & 0,74 & 2,78 & 0,61 & 9341,0 & 0,184 \\
\hline Estrategias de compensación & 2,93 & 0,71 & 3,00 & 0,63 & 9646,0 & 0,363 \\
\hline Estrategias metacognitivas & 2,75 & 0,76 & 2,89 & 0,73 & 9550,5 & 0,299 \\
\hline Estrategias afectivas & 2,41 & 0,64 & 2,57 & 0,72 & 9104,0 & 0,097 \\
\hline Estrategias sociales & 3,04 & 0,85 & 3,10 & 0,73 & 10182,5 & 0,870 \\
\hline \multicolumn{1}{|c|}{ SILL total } & $\mathbf{2 , 7 4}$ & $\mathbf{0 , 6 2}$ & $\mathbf{2 , 8 5}$ & $\mathbf{0 , 5 4}$ & $\mathbf{9 4 9 0 , 0}$ & $\mathbf{0 , 2 6 2}$ \\
\hline Aptitud para las lenguas & 3,66 & 0,25 & 3,36 & 0,38 & 10293,5 & 0,993 \\
\hline Dificultad del aprendizaje de una lengua & 3,19 & 0,62 & 3,25 & 0,55 & 9451,5 & 0,237 \\
\hline Naturaleza del aprendizaje de una lengua & 3,70 & 0,59 & 3,72 & 0,45 & 10184,5 & 0,872 \\
\hline $\begin{array}{l}\text { Estrategias de aprendizaje y de } \\
\text { comunicación }\end{array}$ & 3,51 & 0,44 & 3,42 & 0,39 & 9690,0 & 0,396 \\
\hline Motivaciones y expectativas & 4,23 & 0,51 & 4,15 & 0,50 & 10190,5 & 0,879 \\
\hline BALLI total & $\mathbf{3 , 6 1}$ & $\mathbf{0 , 2 9}$ & $\mathbf{3 , 6 3}$ & $\mathbf{0 , 2 9}$ & $\mathbf{1 0 2 2 7 , 0}$ & $\mathbf{0 , 9 2 0}$ \\
\hline
\end{tabular}

$* \mathrm{p}<.05$

Fuente: Elaboración propia.

Los datos obtenidos a través del test de Spearman revelaron que el uso de las estrategias de memoria se encuentra negativamente correlacionadas con la naturaleza del aprendizaje de una lengua extranjera $(\mathrm{r}=-0,127, \mathrm{p}<.05)$ y con las estrategias de aprendizaje y comunicación $(\mathrm{r}=$ $0,125, \mathrm{p}<.05)$. De igual forma, se encontró una correlación negativa entre las estrategias afectivas y de aprendizaje y comunicación $(r=-0,127, \mathrm{p}<.05)$, lo cual puede observarse en la tabla 4 . Estos resultados son discordantes con los hallazgos de Li (2010), Sioson (2011), Azar y Saeidi (2013), y Hapsari (2016), pues en dichas investigaciones no se encontraron correlaciones negativas coincidentes con las resultantes en el presente estudio. De hecho, en estas investigaciones, se hallaron más correlaciones positivas y las negativas se presentaron en una baja proporción.

Práxis Educativa, Ponta Grossa, v. 16, e2116572, p. 1-17, 2021 Disponível em: <https://www.revistas2.uepg.br/index.php/praxiseducativa> 
El resultado de este estudio significa que posiblemente la disminución del uso de las estrategias de memoria y las estrategias afectivas son influenciadas por las creencias sobre la naturaleza del aprendizaje de una lengua, y por las estrategias de aprendizaje y comunicación. Esta especulación es confirmada por los resultados obtenidos en las medias de las dos creencias que se acaban de mencionar, las cuales fueron altas (véase Tabla 2), en contraste con las puntuaciones de las medias de las dos estrategias del SILL con las que se presentaron las correlaciones ya que mostraron los valores más bajos (véase Tabla 1). En otras palabras, entre mayor grado de acuerdo se presenta en las creencias sobre la naturaleza y las estrategias de aprendizaje y comunicación, menor es el empleo de las estrategias de memoria y estrategias afectivas.

Teniendo en cuenta que los estudios con los que fueron contrastados estos resultados, se realizaron en otros países, podría deducirse que algunas de las variables de los constructos que conforman el SILL y el BALLI se comportan diferente en otros contextos y, quizás, podría atribuirse a las diferencias socioculturales, socioeducativas, socioeconómicas, y a factores individuales tales como la edad, el sexo, el nivel de dominio del idioma, el contexto de aprendizaje, los estilos de aprendizaje, ciertos aspectos cognitivos y afectivos, la personalidad, el tipo de tarea, las creencias y las experiencias previas. Por lo tanto, podría especularse que la selección de las estrategias que hace un estudiante está condicionada a diversos aspectos personales.

Las correlaciones encontradas podrían indicar que esta muestra confía en menor proporción en el uso de estrategias de memoria y estrategias afectivas para el aprendizaje del inglés, a pesar de que las puntuaciones de las medias se concentraron en un rango medio de uso, debido a que ambas estrategias obtuvieron los valores más bajos en las medias (véase Tabla 1). Por otro lado, esto también hace pensar que las creencias sobre la naturaleza de una lengua y las estrategias de aprendizaje y comunicación, probablemente, reducen la frecuencia de uso de las estrategias de memoria y las estrategias afectivas. Llama la atención que, en las creencias correspondientes a la gramática, el vocabulario y la traducción, los resultados indicaron que, para estos estudiantes, dichos aspectos son de gran relevancia, pero las puntuaciones de las estrategias de memoria resultaron estar entre las más bajas, junto con las cognitivas y las afectivas. Se puede pensar entonces que las creencias sobre la naturaleza implican un alto uso de estrategias cognitivas, debido a que estas consisten en acciones concretas para el aprendizaje como la toma de notas, la repetición y la traducción, pero los resultados en esta categoría fueron contradictorios porque, aunque se encuentran en un rango medio de uso, la media mostró estar en el penúltimo lugar con relación a las demás (véase Tabla 1). 
Estrategias de aprendizaje y creencias sobre el idioma inglés: una aproximación correlacional...

Tabla 4 - Correlaciones de Pearson entre el BALLI y el SILL

\begin{tabular}{|c|c|c|c|c|c|c|}
\hline SILL/BALLI & Coeficientes & $\begin{array}{l}\text { Aptitud } \\
\text { para las } \\
\text { lenguas }\end{array}$ & $\begin{array}{c}\text { Dificultad } \\
\text { del } \\
\text { aprendizaje } \\
\text { de una } \\
\text { lengua } \\
\end{array}$ & $\begin{array}{c}\text { Naturaleza } \\
\text { del } \\
\text { aprendizaje } \\
\text { de una } \\
\text { lengua } \\
\end{array}$ & $\begin{array}{l}\text { Estrategias de } \\
\text { aprendizaje y } \\
\text { comunicación }\end{array}$ & $\begin{array}{l}\text { Motivaciones } \\
\text { y expectativas }\end{array}$ \\
\hline \multirow[t]{2}{*}{$\begin{array}{l}\text { Estrategia } \\
\text { Memoria }\end{array}$} & $\begin{array}{c}\text { Coef. de } \\
\text { correlación }\end{array}$ & $-0,110$ & $-0,040$ &,$- 127 *$ &,$- 125^{*}$ & $-0,046$ \\
\hline & Sig. (bilateral) & 0,056 & 0,485 & 0,028 & 0,029 & 0,428 \\
\hline \multirow[t]{2}{*}{$\begin{array}{l}\text { Estrategia } \\
\text { Cognitiva } \\
\end{array}$} & $\begin{array}{c}\text { Coef. de } \\
\text { correlación }\end{array}$ & $-0,031$ & $-0,014$ & $-0,050$ & $-0,041$ & 0,070 \\
\hline & Sig. (bilateral) & 0,594 & 0,811 & 0,387 & 0,480 & 0,221 \\
\hline \multirow[t]{2}{*}{$\begin{array}{c}\text { Estrategia } \\
\text { Compensacion }\end{array}$} & $\begin{array}{c}\text { Coef. de } \\
\text { correlación }\end{array}$ & $-0,108$ & 0,041 & $-0,022$ & $-0,022$ & 0,023 \\
\hline & Sig. (bilateral) & 0,061 & 0,479 & 0,703 & 0,699 & 0,688 \\
\hline \multirow[t]{2}{*}{$\begin{array}{c}\text { Estrategia } \\
\text { Metacognitiva }\end{array}$} & $\begin{array}{c}\text { Coef. de } \\
\text { correlación }\end{array}$ & $-0,062$ & $-0,018$ & $-0,057$ & $-0,059$ & 0,021 \\
\hline & Sig. (bilateral) & 0,284 & 0,749 & 0,322 & 0,309 & 0,719 \\
\hline \multirow[t]{2}{*}{$\begin{array}{l}\text { Estrategia } \\
\text { Afectivas }\end{array}$} & $\begin{array}{c}\text { Coef. de } \\
\text { correlación }\end{array}$ & $-0,047$ & $-0,016$ & $-0,099$ &,$- 121 *$ & $-0,007$ \\
\hline & Sig. (bilateral) & 0,419 & 0,779 & 0,086 & 0,035 & 0,910 \\
\hline \multirow[t]{2}{*}{$\begin{array}{c}\text { Estrategia } \\
\text { Sociales }\end{array}$} & $\begin{array}{c}\text { Coef. de } \\
\text { correlación }\end{array}$ & $-0,076$ & $-0,012$ & $-0,075$ & $-0,066$ & $-0,001$ \\
\hline & Sig. (bilateral) & 0,188 & 0,836 & 0,195 & 0,253 & 0,991 \\
\hline
\end{tabular}

Fuente: Elaboración propia.

\section{Discusión y conclusiones}

En esta investigación se analizaron las estrategias de aprendizaje para el aprendizaje de lenguas (SILL) y las creencias sobre el aprendizaje de una lengua (BALLI), y se examinaron las correlaciones entre ambas variables en una muestra de estudiantes que cursan la asignatura de inglés en los niveles A1 al B1 en el Tecnológico de Antioquia, Institución Universitaria (TdeA - I.U.). La estadística descriptiva indicó que las estrategias de aprendizaje más utilizadas son las sociales. Esto indica que las estrategias que implican la interacción con los demás son las más privilegiadas por estos estudiantes, ya que son de utilidad durante la interacción y promueven la colaboración, permiten que los demás evalúen, expresen opiniones y generan la posibilidad de compartir información. Se evidenció que esta muestra emplea las estrategias de aprendizaje para el estudio del inglés de forma moderada, a consecuencia de que las puntuaciones de todas las medias del SILL se ubicaron en un rango intermedio.

Por otro lado, se encontró que los participantes se encuentran en un rango medio en cuanto a la frecuencia de uso general de las estrategias de aprendizaje $(\mathrm{M}=2.82$ / $\mathrm{DS}=0.69)$. Para AliZarei y Rahmani (2013), las estrategias de tipo afectivo y metacognitivo son los mejores predictores de un aprendizaje efectivo de la lengua extranjera. Es posible considerar que quizás en los niveles de dominio del inglés más altos (B2, C1 y C2), el comportamiento de las relaciones entre el SILL y el BALLI pueda generar un mayor número de correlaciones entre ambas.

Respecto a las creencias sobre el aprendizaje de una lengua, aquellas que conformaron la dimensión "Motivaciones y expectativas", obtuvieron la mayor puntuación $(\mathrm{M}=4.14$ / SD = 
0.69). Esto demuestra que casi la totalidad de los participantes posee una motivación instrumental que los incita a desarrollar la competencia comunicativa en el inglés, puesto que esta les posibilitará obtener oportunidades laborales y ser parte de una comunidad angloparlante de personas.

En lo concerniente a las diferencias de sexo, la prueba U de Mann-Whitney determinó que solo se presentaron diferencias significativas en las estrategias de memoria y no se encontraron diferencias estadísticamente significativas entre hombres y mujeres en las dimensiones del BALLI. Se atribuyen las diferencias sobre empleo de las estrategias de aprendizaje a las habilidades innatas que poseen las mujeres en cuanto a las tareas que requieren memoria y el aprendizaje verbal, de acuerdo con Navarro et al. (2014).

El test de Spearman para correlaciones bivariadas demostró que las estrategias de memoria tienen una correlación negativa entre la dimensión de la naturaleza del aprendizaje de una lengua y las estrategias de aprendizaje y comunicación. Además, se halló una correlación negativa entre las estrategias afectivas y las estrategias de aprendizaje y comunicación. La información obtenida de las correlaciones, junto con los datos descriptivos, podrían hacer pensar que, debido a que se presentaron niveles de acuerdo altos en la dimensión sobre la naturaleza del lenguaje y la dimensión de las estrategias de aprendizaje y comunicación, estos podrían haber causado la disminución en el uso de las estrategias de memoria y de las estrategias afectivas, lo cual se reflejó en las puntuaciones de las medias.

De este estudio se derivan varias implicaciones pedagógicas, las cuales se enuncian a continuación. Es evidente que una de las metas anheladas en el proceso educativo es promover el aprendizaje autónomo de los estudiantes no solo en el aula, sino en las actividades de aprendizaje que se desarrollan fuera de ellas. De este modo, no sería necesario que los discentes soliciten a un facilitador o mediador de manera permanente. Esto entonces hace pensar en la necesidad de utilizar didácticas, métodos y herramientas pedagógicas que fomenten la independencia, la automotivación y la autoevaluación, de tal forma que ellos logren tener control sobre su aprendizaje, aprendan de sus errores, se tracen metas y logren desarrollar las habilidades comunicativas en congruencia con las competencias del nivel de inglés que se encuentran cursando.

El uso de las estrategias de aprendizaje para aprender una lengua extranjera se convierte entonces en los procedimientos o recursos que los estudiantes pueden emplear consciente y organizadamente para aprender, resolver las dificultades que se presentan en dicho proceso y facilitar el aprendizaje. De igual forma, podría pensarse en el docente como un facilitador que ayuda a los estudiantes a analizar sus decisiones, a reflexionar sobre las mismas y, por otro lado, servir como un apoyo psicosocial que intervenga en variables que inciden en el aprendizaje (motivacionales, cognitivas, situaciones familiares, sociales, psicológicas, entre otras). Es de gran importancia considerar, que también se requiere a un docente de inglés con un amplio dominio de la lengua y con una robusta formación pedagógica que permita orientar a los estudiantes, de tal forma que contribuya a allanarles el camino hacia el logro de la competencia comunicativa. Se hizo evidente en este estudio que las creencias que tienen los estudiantes frente al aprendizaje del inglés pueden ser positivas o negativas y comprenden diferentes aspectos.

Dentro de las limitaciones derivadas de este estudio, se manifiesta que es posible que las estrategias de aprendizaje y las creencias sobre el aprendizaje del inglés puedan estar influenciadas por variables como la edad, la nacionalidad, la carrera que se está cursando, el estrato social, aspectos socioculturales, experiencias previas, el auto concepto, la autonomía, entre otras. Por lo tanto, se podrían incluir en otros estudios variables que aporten información importante para comprender los aspectos que causan mayor influencia en el uso de las estrategias de aprendizaje, y si las creencias se transforman con relación a dichas variables o si permanecen estáticas. Por otro lado, se considera que los instrumentos SILL y BALLI podrían ser enriquecidos por otras

Práxis Educativa, Ponta Grossa, v. 16, e2116572, p. 1-17, 2021 Disponível em: <https://www.revistas2.uepg.br/index.php/praxiseducativa> 
herramientas que permitan recolectar información, tales como diario de campo, entrevistas, entre otros.

Finalmente, como líneas futuras de investigación, se propone lo siguiente: 1. considerar las creencias que otros investigadores han encontrado y las que puedan surgir en las instituciones educativas privadas y públicas, y de este modo generar nuevos inventarios provenientes del contexto colombiano, después de profundizar en la identificación de la génesis de cada una de ellas. 2. realizar estudios que incluyan variables individuales como la actitud, la autonomía, las estrategias de metacognición, la autorregulación, y la metodología, entre otras, lo cual podría ampliar el espectro de los factores que inciden en el proceso de aprendizaje de una lengua extranjera.

\section{Referencias}

AHMADI, H.; ABDOLLAHZADEH, P.; TAGHINEZHAD, A.; MOHAMMAD, A. The relationship between beliefs about language learning, gender, and the use of language learning strategies of Iranian EFL learners. Modern Journal of Language Teaching Methods (MJLTM), v. 6 , n. 5, p. 31-4, 2016.

ALCALDE, N. Principales métodos de enseñanza de lenguas extranjeras en alemania. Revista de Lingüística y Lenguas Aplicadas, n. 6, p. 9-23, 2011. DOI: http://dx.doi.org/10.4995/rlyla.2011.878

ALI-ZAREI, A.; RAHMANI, H. The relationship between Iranian EFL learners' beliefs about language learning and language learning strategy use. Journal on English Language Teaching, v. 28, p. $1-10,2013$.

AZAR, F. K.; SAEIDI, M. The relationship between Iranian EFL learners' beliefs about language learning and their use of learning strategies. English Language Teaching, v. 6, n. 11, p. 167174, 2013. DOI: https://doi.org/10.5539/elt.v6n11p167

BARRIOS, E.; MONTIJANO, M. P. Uso declarado de estrategias y su variación según el nivel de competencia en aprendientes universitarios de inglés como lengua extranjera. Revista Signos Estudios de Linguística, v. 50, n. 95, p. 312-336, 2017. DOI: https://doi.org/10.4067/s0718$\underline{09342017000300312}$

CEN, H. Research on adult English learning model and implementation mechanism based on wisdom learning. Studies in Literature and Language, v. 19, n. 3, 33-38, 2019. DOI: https://doi.org/10.3968/11425

CHANG, C-Y.; SHEN, M-C. The effects of beliefs about language learning and learning strategy use of junior high school EFL learners in remote districts. Research in Higher Education Journal, v. 8, p. 1-8, 2010.

CONSEJO DE EUROPA. Common European Framework of Reference for Languages: learning, teaching, assessment (CEFR). Cambridge: Cambridge University Press, 2001.

DAIF-ALLAH, A. Beliefs about foreign language learning and their relationship to gender. English Language Teaching, v. 5, n. 10, p. 20-33, 2012. DOI: http://dx.doi.org/10.5539/elt.v5n10p20 
DEMIR, Y.; MUTLU, G.; SISSMAN, Y. S. Exploring the oral communication strategies used by Turkish EFL learners: a mixed methods study. International Journal of Instruction, v. 11, n. 2, p. 539-554, 2018. DOI: https://doi.org/10.12973/iji.2018.11237a

FEBRIANI, N.T. Correlation between metacognitive strategy, foreign language aptitude and motivations in language learning. Jurnal Bahasa Lingua Scientia, v. 9, n. 2, p. 357-370, 2017. DOI: https://doi.org/10.21274/ls.2017.9.2.357-370

FELECIYA, J. N.; ZAFAR, S.; KHAN, Z. The influence of language learning strategies on language related employability skills. Asia Pacific Journal of Research, v. 1, n. 33, p. 183-191, 2015.

FRANCO, P.; PINO, M.; RODRÍGUEZ, B. Tipología y frecuencia del uso de estrategias en el aprendizaje del inglés como lengua extranjera. Enseñanza \& Teaching, v. 27, n. 2, p. 171-191, 2009.

GARCÍA HERRERO, M. D. M. Diferencias en el uso de estrategias en el aprendizaje de la lengua extranjera según el género. Revista de Investigación Educativa, v. 31, n. 1, p. 61-80, 2012.

GÓMEZ, J. F. Creencias sobre el aprendizaje de una lengua extranjera en el contexto universitario. Íkala, Revista de Lenguaje y Cultura, v. 22, n. 2, p. 203-219, 2017. DOI: https://doi.org/10.17533/udea.ikala.v22n02a03

GÓMEZ, J. F. Diferencias en las creencias entre hombres y mujeres acerca del aprendizaje del idioma inglés. Revista Signos Estudios de Linguística, v. 51, n. 97, p. 193-213, 2018. DOI: https://doi.org/10.4067/S0718-09342018000200193

HAKAN, K.; BALYER, A.; ALCI, B. An investigation of undergraduates' language learning strategies. Procedia - Social and Behavioral Sciences, v. 197, p. 1348-1354, 2015. DOI: https://doi.org/10.1016/j.sbspro.2015.07.388

HAPSARI, P. A. The correlation between belief and students' language learning strategies at English department of UNESA. Retain, v. 4, n. 1, p. 1-9, 2016.

HAYATI, N. A study of English language learning beliefs, strategies, and English academic achievement of the ESP students of STIENAS Samarinda. Journal of Education, v. 15, n. 2, p. 297-323, 2015. DOI: https://doi.org/10.21093/di.v15i2.211

HERRERA, Á. M. Las estrategias de aprendizaje. Innovación y Experiencias Educativas, n. 16, p. 1-14, 2009.

HORWITZ, E. K. Surveying student beliefs about language learning. In: WENDEN, A.; RUBIN, J. (ed.). Learner strategies in language learning. Nova Jersey: Prentice-Hall International, 1987. p. 119-129.

KOVACEVIC, E.; AKBAROV, A. Language learning beliefs and strategies: A Bosnian EFL case. Epiphany: Journal of Transdisciplinary Studies, v. 8, n. 3, p. 74-87, 2015. DOI: http://dx.doi.org/10.21533/epiphany.v8i3.178

LI, F. Relationship between EFL learners' belief and learning strategy use by English majors in vocational colleges. Journal of Language Teaching and Research, v. 1, n. 6, p. 858-866, 2010. DOI: https://doi.org/10.4304/jltr.1.6.858-866 
LIGHTBOWN, P. M.; SPADA, N. How languages are learned. Oxford Handbooks for Language Teachers. 4th ed. Oxford: University Press, 2013.

LÓPEZ RUA, P. The sex variable in foreign language learning: An integrative approach. Porta Linguarum, v. 6, p. 99-114, 2006. DOI: https://doi.org/10.30827/digibug.30663

MESHKAT, M.; SAEB, F. The relationship between high school students' beliefs about language learning and their use of language learning strategies. Issues in Language Teaching, v. 1, n. 2, p. 273-296, 2012.

MOSLEHIFAR, M. A.; IBRAHIM, N. A. English language oral communication needs at the workplace: feedback from human resource development (HRD) trainees. Procedia - Social and
Behavioral
Sciences,
n.
66
p.
529-536,
2012.
DOI:

https://doi.org/10.1016/j.sbspro.2012.11.297

MUDRA, H. Prospective EFL teachers beliefs about language learning and gender differences in a higher education context. International Journal of Academic Research in Education, v. 2, n. 1, p. 42-50, 2016. DOI: https://doi.org/10.17985/ijare.13474

NAHAVANDI, N.; MUKUNDAN, J. Language learning strategy use among Iranian Engineering EFL learners. Advances in Language and Literary Studies, v. 5, n. 5, p. 34-45, 2014. DOI: https://doi.org/10.7575/aiac.alls.v.5n.5p.34

NAVARRO, E.; CALERO, M. D.; CALERO-GARCÍA, M. J. Diferencias entre hombres y mujeres mayores en funcionamiento cognitivo y calidad de vida. European Journal of Investigation in Health, Psychology and Education, v. 4, n. 3, p. 267-277, 2014. DOI: https://doi.org/10.30552/ejihpe.v4i3.79

ORREGO, L. M.; DÍAZ, A. E. Empleo de estrategias de aprendizaje de lenguas extranjeras: inglés y francés. Íkala, Revista de Lenguaje y Cultura, v. 15, n. 1, p. 105-142, 2010.

ORTEGA, L. Understanding second language acquisition. New York: Routledge, 2013.

OXFORD, R. Language learning strategies. What every teacher should know. Boston/Massachusetts: Heinle \& Heinle Publishers, 1990.

OXFORD, R; BURRY-STOCK, J. Assessing the use of language learning strategies worldwide with the ESL/EFL version of the strategy inventory for language learning (SILL). Pergamon, v. 23, n. 1, p. 1-23, 1995. DOI: https://doi.org/10.1016/0346-251X(94)00047-A

PAŠALIĆ, M. Learning more FLs, learning English outside of school and gender correlated with success, beliefs and language learning strategies. Folia Linguistica ett Litteraria, v. 21, n. 1, p. 177-193, 2018. DOI: https://doi.org/10.31902/fll.21.2018.8

RISUEÑO, J. J.; VÁZQUEZ, M. L.; HIDALGO, J.; DE LA BLANCA, S. Language learning strategy use by Spanish EFL students: The effect of proficiency level, gender, and motivation. Revista de Investigacion Educativa, v. 34, n. 1, p. 133-149, 2016. DOI: https://doi.org/10.31902/fll.21.2018.8

SIOSON, I. Language learning strategies, beliefs, and anxiety in academic speaking task. PHILIPPINE ESL Journal, v. 7, p. 3-27, 2011. 
TRI FEBRIANI, N. Correlation between metacognitive strategy, foreign language aptitude and motivations in language learning. Jurnal Bahasa Lingua Scientia, v. 9, n. 2, 357-370, 2017. DOI: https://doi.org/10.21274/1s.2017.9.2.357-370

TUGRUL, Ç. Guessing the meanings of words from context: why and how. International Journal of Applied Linguistics \& English Literature, v. 1, n. 6, p. 177-181, 2012. DOI: https://doi.org/10.7575/ijalel.v.1n.6p.177

TUGRUL, C.. The Grammar-translation method and the use of translation to facilitate learning in ESL classes. Journal of Advances in English Language Teaching, v. 1, n. 4, p. 103-105, 2013.

WAHDAH, N.; AININ, M.; HAMID, M. A. Dayakese students' beliefs about Arabic language learning and their relation with the language learning strategies. Journal of Arabic Linguistics and Education, v. 4, n. 1, p. 1-18, 2018. DOI: https://doi.org/10.28918/alsinatuna.v4i1.1589

ZHOU, Y. English language learning strategy use by Chinese senior high school students. English Language Teaching, v. 3, n. 4, p. 152-159, 2010.

Recibido: 30/08/2020

Versión corregida recibida: 16/02/2021

Aceptado: 17/02/2021

Publicado online: $18 / 02 / 2021$ 